\title{
Raising nursing student awareness of how it feels to have dementia with the use of $360^{\circ}$ video
}

\author{
Kristine Newman, Parmeet K. Kahlon, Shujie Li \\ Daphne Cockwell School of Nursing, Ryerson University, Toronto, Canada
}

Received: June 22, 2020

Accepted: September 26, $2020 \quad$ Online Published: October 18, 2020

DOI: $10.5430 /$ jnep.v11n2p7

URL: https://doi.org/10.5430/jnep.v11n2p7

\begin{abstract}
Objective: This paper aims to look at raising nursing student awareness of how it feels to have dementia through the use of 360-degree video. We were particularly interested in raising awareness amongst nursing students perceptions of the behavioral and psychological symptoms of dementia to promote holistic care provision guided by an empathetic understanding of how it feels to have dementia.

Methods: We used a mixed methods approach to investigate nursing students' awareness of how it feels to have dementia through the use of the 360-degree videos originally developed for a creative new media arts-exhibit at Toronto Rehabilitation Institute for raising dementia awareness. Data was collected quantitatively and qualitatively with thematic and content analyses. A focus group was conducted to explore participants' awareness of how it feels to have dementia by viewing the 360-degree video, empathy towards people with dementia, and knowledge of dementia.

Results: The 360-degree video is an arts-based knowledge-translation strategy that was used to raise nursing students' awareness on behavioural and psychological symptoms of dementia. While the majority of the participants had some levels of awareness and understanding of dementia prior to the viewing of 360-degree video, they were nonetheless challenged by the content of the 360-degree video that led to new perspectives on dementia.

Conclusions: The three themes identified in the thematic analysis of the focus group are understanding dementia from a new perspective, embodiment of dementia experience, and self-reflection and practice changes. These themes reflect the usefulness of 360-degree video as an arts-based knowledge translation strategy in raising dementia awareness in nursing students.
\end{abstract}

Key Words: Arts-based knowledge translation, Virtual reality, Behavioral and psychological symptoms of dementia (BPSD), Dementia, Education, Communication

\section{INTRODUCTION}

With an aging population, there has been a steady increase in the incidence of neurodegenerative conditions, like dementia, that further complicate healthcare delivery. ${ }^{[1]}$ Persons with dementia experience unique behavioral and psychological symptoms of dementia (BPSD), including agitation, irritability, and confusion, which differ among the sexes. ${ }^{[2]}$ This contributes to a heightened need for healthcare provider education related to dementia, to ensure the provision of sensitive, evidence-informed care.

Despite the push towards using research evidence as a rationale for clinical care, there is a persistent evidence-practice gap that prevents adequate uptake and application of research evidence in everyday practice. ${ }^{[3]}$ To help mitigate this disparity, there has been an emergence of arts-based knowledge

\footnotetext{
* Correspondence: Kristine Newman; Email: kristine.newman@ ryerson.ca; Address: Daphne Cockwell School of Nursing, Ryerson University, Toronto, Canada.
} 
translation (ABKT) as a novel method of research dissemination that utilizes creative media to ensure information comprehension across a diverse audience. ${ }^{[4]}$ Different art forms, including video, installation art, visual arts and images have been used as ABKT mediums, with success in improving viewer understanding and uptake of evidence. ${ }^{[5]}$

This study aims to understand nursing students' awareness of how it feels to have dementia through the use of 360degree video. Videos were produced based on a separate research study, and fictitiously constructed and filmed in two narratives based on research themes. These videos were then shown to the participants using Oculus 360-degree video technology, with an aim to understand how they contribute to increasing awareness, empathy, and knowledge amongst users towards people with dementia.

The 360-degree videos are available for viewing at the links mentioned below:

1) Nina: https://drive.google.com/file/d/1sp97s jsGd_RZbvIjLY149U1r2NilMhVz/view?usp=sharing

2) Charles: https://drive.google.com/open?id=1sj d68JfXG6uV85ZvxDLcfwckf5bIyyNX

For more information on the 360 video development, please refer to Impact of EMBODY Experiencing Dementia through New Media Exhibit: Engaging nursing students and the public about dementia through arts-based knowledge translation methods. ${ }^{[6]}$ The exhibit featured multiple art and digital media forms including photographs, paintings, written word, data visualizations, augmented and virtual reality (e.g. 360degree video).

\section{LiteratURE REVIEW}

An aging population presents several healthcare challenges that are further complicated by clinical syndromes such as dementia. Caused by progressive neurodegeneration, dementia typically presents with neuropathological conditions such as Alzheimer's disease and vascular infarctions. ${ }^{[7]}$ The resulting changes in brain physiology contribute to a host of behavioral and psychological symptoms of dementia (BPSD) including agitation and memory loss. ${ }^{[2]}$

These BPSD create significant stress for persons with dementia, as well as their caregivers. ${ }^{[8]}$ The literature mentions that there is a significant association between caregiving for persons with dementia, psychological and physical health issues. ${ }^{[9]}$ Unfortunately, traditional therapies, such as cognitive behavioral therapy and group therapy, have not been found to be effective in reducing caregiver anxiety. ${ }^{[10]}$ To alleviate this stress, there is an urgent need to identify and resolve BPSD in a timely manner, allowing people with dementia and their caregivers to lead higher quality lives.

A systematic review revealed that there is a significant lack of confidence and knowledge in nursing professionals when providing care for persons with dementia. ${ }^{[1]}$ Nursing students also report this difficulty in providing holistic, informed dementia care. ${ }^{[12]}$ These areas of difficulty can be partially attributed to a lack of dementia-focused education and experiences in clinical settings, as well as a prominent evidencepractice gap, as research findings are not consistently incorporated into healthcare practice. ${ }^{[12,13]}$ A lack of empathetic understanding of what it means to have dementia has been cited as a primary cause of this gap. ${ }^{[14]}$ This gap can have serious consequences, as it potentially denies vulnerable populations the appropriate, evidence-informed care they require.

Over the years, ABKT has emerged as an alternative method for evidence dissemination. Incorporating the use of different media, such as poetry, video and images, ABKT uses circular methods of information dissemination that are unique from the traditionally linear approaches adopted by academic articles. ${ }^{[13]}$ It has been well developed in the literature that ABKT can evoke affective responses, construct alternative forms of representation that promote dialogue, shared storytelling, and reflection, and engage audiences on emotive, embodied, and intellectual levels. ${ }^{[15]}$ Research has shown that creative knowledge translation through the use of dementia simulations has proven to be successful in improving content retention and empathy in nursing students, making it a key area to focus on and refine. ${ }^{[12]}$

Virtual reality is an emerging technology that is being adapted to several uses. According to the literature, 360degrees videos are a form of virtual reality that has been proven to cause changes in behaviors in attitudes, likely related to the deeply immersive experiences they provide. ${ }^{[16,17]}$ The use of these technologies allows individuals to explore the situation according to their interests, and fully experience the sights and sounds associated with the scenario that help to better convey emotions. ${ }^{[18]}$ The videos used for the study were from the point of view of a person with dementia, with the use of editing technology to create agitation and perceptual distortions that are typically experienced with dementia. ${ }^{[6]}$ By viewing these videos with the 360-degree video technology, an immersive experience of what it feels to have dementia was created.

The use of virtual reality training allows students to look beyond their goal of clinical treatment provision to consider the emotions and feelings of the person with dementia, and thus provide patient-centered care. ${ }^{[19]}$ This is in line with 
findings from the literature, where it was seen that the use of virtual reality technology in dementia education attributed to a three times improvement in empathy and understanding. [20] Students benefited from being able to explore uncommon, yet highly significant clinical situations. ${ }^{[21]}$ By utilizing 360degree immersive videos from male and female perspectives, the study aimed at cultivating a deeper awareness of the BPSD, alongside prominent sex differences, in users, as they experienced, first-handedly, the challenges that persons with dementia face. ${ }^{[6]}$ This ultimately helps to reduce caregiver stress and burden, and contributes to better care provision for the person with dementia. ${ }^{[22,23]}$

\section{MethodS}

\subsection{Research design}

A mixed-methods approach was taken to understand nursing students' awareness of how it feels to have dementia by viewing the 360-degree video. Recruited participants were gathered on February 8, 2019 at Ryerson University to view the 360-degree video and participate in the focus group. Preceding the focus group, an introduction on the 360-degree video was presented.

Demographic surveys were collected along with their experiences with dementia. During the focus group, the discussions were led by two interviewers with audio recording collected for the purpose of developing the study transcript. Audio recordings and transcripts were kept confidential and the study procedures were approved by the research ethics board at Ryerson University. The transcripts were coded and thematically analysed by the research team. All participants identifying information were removed from the analysis.

\subsection{Sampling procedure}

Nursing students from the Daphne Cockwell School of Nursing were recruited via electronic and physical distributions of the focus group workshop poster. The poster consisted of a summary of the focus group activity, location, date, time and contact information of the research team. The electronic distribution was made possible by the Scholarly, Research and Creative (SRC) knowledge translation event coordinator. This coordinator posted the focus group event on the news and events page of the Ryerson Faculty of Community Services website. Participant consent forms containing additional information on the study purpose and procedures were sent to students who had contacted the SRC event coordinator regarding their interest for participation. The inclusion criteria for this paper were verbal and written English comprehension and nursing students aged 18 years or above.

\subsection{Measures}

The primary data collection method was a focus group conducted on February 8, 2019 at Ryerson University. Eleven open-ended questions for the focus group were developed based on findings in recent literature regarding the outcomes of using 360-degree video. Examples of the focus group questions consisted of participants' familiarity with dementia, thoughts on the use of technology to convey information and emotions during the viewing of the 360-degree video (see table 1 for the questionnaire set). All focus group participants completed a demographic information survey that collected information on age, sex, educational level, occupation, and familiarity with dementia. The focus group was audio recorded and the data was transcribed into text format. The transcribed data was then analyzed via thematic analysis. Participant confidentiality was maintained via deidentification of the transcript notes.

Table 1. Focus group questionnaires

\begin{tabular}{|cl}
\hline Questions \\
\hline 1. What does dementia mean to you? \\
2. Did the video make you feel any emotions or think of any specific thoughts? \\
3. How do you feel about people with dementia after watching the 360-degree video? \\
4. Do you feel like your perception changed now that you have seen the 360-degree video? \\
5. How did the 360-degree video help your understanding of dementia? \\
6. What word would you use to describe the 360-degree video experience? \\
7. Did the audio aspect of the video make a difference in your viewing experience? \\
8. Do you perceive watching the 360-degree video as a positive or negative experience? \\
9. Do you feel like the way you act or behave will change based on this EMBODY experience? \\
10. Do you prefer the 360-degree video over flat screen video? \\
11. What behaviours or what things would you do or stop doing because of this video?
\end{tabular}




\section{Results}

A total of 6 participants were recruited for the focus group. All participants were females with education greater than high school diploma. Five of the 6 participants were senior nursing students between the ages of 18-23. The remaining one participant was excluded from the analysis of results as this person did not meet the inclusion criteria for the paper.

All nursing students had some degree of familiarity with dementia either from personal experience or clinical experience. Coding and thematic analysis of the interview transcripts were undertaken by four members of the research team asynchronously followed by episodic group discussions. Three main themes emerged from the data were (1) understanding dementia from a new perspective, (2) embodiment of dementia experience, (3) self-reflection and practice changes. After additional attempts at identifying other codes and themes from 5 participants, all four members of the research team agreed that data saturation was reached.

\subsection{Understanding dementia from a new perspective}

This theme primarily captured the emotional awareness and knowledge of dementia among the participants. First of all, all participants expressed familiarity with dementia as a neurocognitive disorder. Most of their dementia experiences came from their previous practicum and personal experiences. These experiences ranged from knowing the stories of someone with dementia, being a caregiver for family members with dementia, to working as a student in nursing homes, retirement facilities and tertiary care centres.

When participants were asked about the behavioural and psychological symptoms of dementia, terminologies such as confusion, memory loss, cognitive decline, and decreased functional ability were frequently used to describe the condition. In following up with this question, students were also asked about how the 360-degree video affected their existing understanding of dementia. It appears that the use of 360-degree video provoked a deeper awareness among the students. For instance, one participant stated, "I think when I thought of dementia before it was just like memory loss, but now it is like no. They are still this human but stuck inside of this and they just can't get out. So it's like they are still who they were, it's just harder for them to interact with the world now". Another participant stated, "I guess I'd look at it now less of a character change, more of an inability to kind of create those connections, to be able to maneuver yourself in this new situation". For these participants, their preconceived understanding of dementia was limited to the biological and physiological symptoms of dementia. However, the 360-degree video provided the participants with a new form of experiential learning that was corresponded with a more humanistic understanding of dementia.

\subsection{Embodiment of dementia experience}

This theme primarily captured the physical, psychological, and emotional reactions from participants towards patients living with dementia in the 360-degree video. The video provided participants with a useful clinical perspective on how it feels to be patients with dementia. This perspective was meant to facilitate the embodiment of the lived experiences of persons with dementia by constructing a live and virtual day in a life scenario for the participants.

The terms frequently used by participants in response to the 360 experiences were anxious, sad, uncomfortable, and vulnerable. For example, one participant stated, "it really made you have the experience of what it feels like to be that person, not see that person but actually be in that person's shoe". In response to this statement, a student said, "it was like no escape, like you just had to go through what you're seeing and you really felt like you were them".

One participant noted the audio aspect of the 360-degree video helped to create a more immersive experience. This participant stated, "I felt like there was so much (auditory) stimuli going on in the video, so that just made it feel more real". More specifically, the subtitle environmental noises and voices projected directly at the viewer helped to construct a more immersive and realistic experience.

Some participants reported both emotional and visceral responses while viewing the 360-degree video. For example, one student stated, "it made me feel very anxious, my heart kind of started racing because I kind of felt her emotions, like she does not want to be here and it made me in her shoes. I did not want to be here either". The majority of participants preferred the 360-degree video over flat screen video when asked how the 360-degree aspect of the video influenced their experience. As one participant stated, "it was different because I could just look away".

\subsection{Self-reflection and practice changes}

This theme is focused on self-reflection and practice changes towards dementia care. All five students spoke about their past clinical experiences with patients living with dementia. Their lookback on their caregiver experiences were associated with desires for practice changes. One participant stated, "I see them like every single day, so you forget maybe I should introduce myself again, maybe they forgot who I am". Echoed by this statement, another student said, "it might just ease their anxiety by saying I am the nursing student who is working with you instead of thinking that they will be fine if I just go on with the care tasks". These students expressed varying levels of desire to go back in time and change the 
way they interacted with their patients. However, some students were uncertain on how practices can be changed. In one statement made by a student, "what do I do because these are literally the people that I see everyday. We try to redirect to their anxiety because it is what we have been told, but I am not so sure now". For this particular student, she saw the use of redirection and distraction by nurses to deal with behavioural and psychological symptoms of dementia in the 360-degree video. However, she was situated in the patient's perspective and embodied the experience of a care recipient instead of a caregiver. This change in role made her to think about the effectiveness of behavioural strategies for BDSP from the perspective of the patient.

\subsection{Limitations}

We targeted nursing students to look at the impact of 360degree video on raising awareness of the behavioural and psychological symptoms of dementia. All participants were recruited from Ryerson University, of which all participants were females between the ages of 18-23. The interviews were recorded and transcripted for thematic analysis. It should be noted that a more broadly targeted demographic group would probably lead to different observations and study outcomes. This small grouping of participants also makes the study results difficult to generalize beyond the studied cohort. However, this research does add to the existing literature on the possible application of virtual reality as a form of artsbased knowledge translation strategy to facilitate dementia awareness knowledge translation. It is recommended that further research be conducted on a larger sample of nursing students from different academic institutions or professions. This expanded sample should include students presenting more diverse sexual orientations, gender, and educational backgrounds. Moreover, the larger qualitative data set should encompass an examination of participants' previous virtual reality experiences as this can influence their experiences and study outcomes.

\section{Discussion}

In this study of nursing students' awareness of how it feels to have dementia by viewing the 360-degree videos, understanding dementia from a new perspective, the embodiment of dementia experience, and self-reflection and practice changes emerged as three central themes in the focus group. The submergence within virtual reality served as a medium for the embodiment of dementia to manifest. All participants reported varying degrees of cognitive and emotional responses. One participant even had visceral responses described as racing heart rate and anxiety-provoking. Almost all participants had a newfound perspective on the lived experiences of persons with dementia. These perspectives were often de- scribed as self-realization of how fixated their preconceived ideas of dementia were. More specifically, dementia was no longer associated with straightforward labels of memory loss and social dysfunction, but rather a condition with physical, psychosocial, and emotional implications.

The participants' understanding of dementia from a new perspective, embodiment of their experience, and self-reflection for their practice were important catalysts to their stimulated awareness of how it feels to have dementia among the participants. These catalysts were made possible by having the students simulate the experience of dementia via the perspective of the care recipient. This awareness can be applied to their practice as a nursing student to better appreciate the person with dementia's perspective. With the increasing number of the Canadian population moving into the senior category, it is expected to see a significant rise in the number of patients with dementia. This growing health trend will likely be corresponded with increased demand for specialized nursing care focused on the behavioural and psychological symptoms of dementia. In terms of implications for practice, the 360-degree video may be used for nursing students as a simulation knowledge translation strategy to facilitate the development of dementia care knowledge. It would be beneficial to incorporate EMBODY 360-degree videos in the nursing geriatric and dementia curriculum. For nurses working with the geriatric population, the 360-degree video may also be used as a quality assurance tool for the purpose of maintaining their nursing knowledge and skill competencies related to dementia care.

\section{Conclusion}

The application of 360-degree video or virtual reality as a form of arts-based knowledge translation strategy for the purpose of dementia education is a limited area of research in the current nursing education literature. Since pathophysiology, clinical manifestations and implications of dementia are multidimensional; it is often challenging to translate existing research into clinical knowledge. However, the 360-degree video offers an opportunity for learners to 'live with dementia' by simulating and situating the learner within the first point of view of the care recipient. This useful method of learning was able to evoke visceral, cognitive, psychosocial and emotional reactions in study participants that were associated with improved empirical and aesthetic knowings of dementia. We also recommend that 360-degree videos such as EMBODY are regularly used in nursing curriculum

\section{ACKNOWLEDGEMENTS}

We would like to thank our participants for their time and contributions towards this study. Also, we appreciate the ef- 
forts of the Daphne Cockwell School of Nursing SRC event coordinator to help recruit participants. We would like to especially thank Jacky Au Duong for his support and guidance throughout the study. We would also like to acknowledge the developers of the 360-degree videos. We are grateful to the Research Ethics Board at Ryerson University. We would also like to acknowledge the gracious funding contributions from Ryerson University through the Ryerson Creative Fund, the Faculty of Community Services publication funding, and the Alzheimer Society Research Program (ASRP) grant for video development.

\section{CONFLicts OF InTEREST Disclosure}

The authors declare that there is no conflict of interest.

\section{REFERENCES}

[1] GBD 2016 Dementia Collaborators. Global, regional, and national burden of Alzheimer's disease and other dementias, 1990-2016: a systematic analysis for the Global Burden of Disease Study 2016. The Lancet Neurology. 2019; 18(1): 88-106. https://doi .org/ 10.1016/S1474-4422(18)30403-4

[2] Chakraborty S, Lennon JC, Malkaram SA, et al. Serotonergic system, cognition, and BPSD in Alzheimer's disease. Neuroscience Letters. 2019; 704: 36-44. PMid:30946928 https://doi.org/10.1016/ j.neulet.2019.03.050

[3] Hickman LD, Digiacomo M, Phillips J, et al. Improving evidence based practice in postgraduate nursing programs: A systematic review. Nurse Education Today. 2018; 63: 69-75. PMid:29407264 https://doi.org/10.1016/j.nedt.2018.01.015

[4] Rieger K, Schultz AS. Exploring arts-based knowledge translation: sharing research findings through performing the patterns, rehearsing the results, staging the synthesis. Worldviews on EvidenceBased Nursing. 2014 May; 11(2): 133-9. PMid:24597543 https: //doi.org/10.1111/wvn.12031

[5] Lapum J, Liu L, Church K, et al. Knowledge translation capacity of arts-informed dissemination: A narrative study. Art/Research International: A Transdisciplinary Journal. 2016; 1(1): 258-282. https://doi.org/10.18432/R2BC7H

[6] Newman K, Duong JA, Kahlon P, Li SJ. Impact of EMBODY Experiencing Dementia through New Media Exhibit: Engaging nursing students and the public about dementia through arts-based knowledge translation methods. Journal of Nursing Education and Practice. 2020; 10(100). https://doi.org/10.5430/jnep.v10n5p100

[7] Barnes LL, Lamar M, Schneider JA. Sex differences in mixed neuropathologies in community-dwelling older adults. Brain Research. 2019; 1719: 11-6. PMid:31128096 https://doi .org/10.1016/ j.brainres. 2019.05.028

[8] Mccabe M, You E, Tatangelo G. Hearing their voice: a systematic review of dementia family caregivers' needs. The Gerontologist. 2016; 56(5). PMid:27102056 https://doi .org/10.1093/gero nt/gnw078

[9] Gilhooly KJ, Gilhooly MLM, Sullivan MP, et al. A meta-review of stress, coping and interventions in dementia and dementia caregiving. BMC Geriatrics. 2016; 16(1). PMid:27193287 https://doi.org/ $10.1186 / \mathrm{s} 12877-016-0280-8$

[10] Hopkinson MD, Reavell J, Lane DA, et al. Cognitive behavioral therapy for depression, anxiety, and stress in caregivers of dementia patients: a systematic review and meta-analysis. The Gerontologist. 2019; 59(4). PMid:29529290 https://doi.org/10.1093/gero $\mathrm{nt} / \mathrm{gnx} 217$

[11] Evripidou M, Charalambous A, Middleton N, et al. Nurses' knowledge and attitudes about dementia care: Systematic literature review. Perspectives in Psychiatric Care. 2019; 55(1): 48-60. PMid:29766513 https://doi.org/10.1111/ppc. 12291

[12] Mastel-Smith B, Kimzey M, He Z. Dementia care education for nursing students. Journal of Nursing Education. 2019Jan; 58(3): 136-43.
PMid:30835800 https : //doi . org/10.3928/01484834-20190 221-03

[13] Scott SD, Brett-MacLean P, Archibald M, et al. Protocol for a systematic review of the use of narrative storytelling and visualarts-based approaches as knowledge translation tools in healthcare. Systematic Reviews. 2013; 2(1): 19. PMid:23514237 https : //doi.org/10.1186/2046-4053-2-19

[14] Dupuis SL, Mitchell GJ, Jonas-Simpson CM, et al. Igniting transformative change in dementia care through research-based drama. The Gerontologist. 2016; 56(6): 1042-52. PMid:26185158 https : //doi.org/10.1093/geront/gnv062

[15] Hall A, Furlong B, Pike A, et al. Using theatre as an arts-based knowledge translation strategy for health-related information: a scoping review protocol. BMJ Open. 2019; 9(10). PMid:31666277 https : //doi.org/10.1136/bmjopen-2019-032738

[16] Chittaro L, Sioni R, Crescentini C, et al. Mortality salience in virtual reality experiences and its effects on users' attitudes towards risk. International Journal of Human-Computer Studies. 2017; 101: 10-22. https://doi.org/10.1016/j.ijhcs.2017.01.002

[17] Schöne B, Wessels M, Gruber T. Experiences in virtual reality: a window to autobiographical memory. Current Psychology. 2017; 38(3): 715-9. https://doi.org/10.1007/s12144-017-9648-y

[18] Shin D, Biocca F. Exploring immersive experience in journalism. New Media \& Society. 2018; 20(8): 2800-23. https ://doi .org/ $10.1177 / 1461444817733133$

[19] Adefila A, Graham S, Clouder L, et al. myShoes - the future of experiential dementia training? The Journal of Mental Health Training, Education, and Practice. 2016; 11(2): 91-101. https: //doi.org/10.1108/JMHTEP-10-2015-0048

[20] McCabe M. Dementia education with and without virtual reality (VR) experiences to increase knowledge, build empathy, change attitude and improve care practice. Alzheimer's \& Dementia: The Journal of the Alzheimer's Association. 2018; 14(7): 185-185. https://doi.org/10.1016/j.jalz.2018.06.2017

[21] Foronda CL, Hudson KW, Budhathoki C. Use of virtual simulation to impact nursing students' cognitive and affective knowledge of evidence-based practice. Worldviews on Evidence-Based Nursing. 2017; 14(2): 168-70. PMid:28207994 https://doi .org/10.111 1/wvn. 12207

[22] Wijma EM, Veerbeek MA, Prins M, et al. A virtual reality intervention to improve the understanding and empathy for people with dementia in informal caregivers: results of a pilot study. Aging \& Mental Health. 2017Oct; 22(9): 1121-9. PMid:28691861 https://doi.org/10.1080/13607863.2017.1348470

[23] Jütten LH, Mark RE, Janssen BWJM, et al. Testing the effectivity of the mixed virtual reality training Into Dementia for informal caregivers of people with dementia: protocol for a longitudinal, quasi-experimental study. BMJ Open. 2017; 7(8). PMid:28827242 https://doi.org/10.1136/bmjopen-2016-015702 